\title{
Nonlinear Receding Horizon Control: Stability without Stabilizing Constraints
}

\author{
Johan Löfberg \\ Division of Automatic Control \\ Department of Electrical Engineering \\ Linköpings universitet, SE-581 83 Linköping, Sweden \\ WWW: http://www. control.isy.liu.se \\ Email: johanl@isy.liu.se
}

June 18, 2001

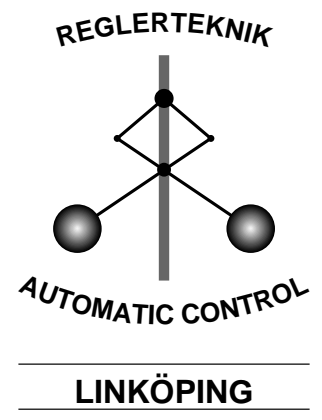

Report No.: LiTH-ISY-R-2356

Submitted to ECC2001

Technical reports from the Automatic Control group in Linköping are available by anonymous ftp at the address ftp.control.isy.liu.se. This report is contained in the file 2356.pdf. 


\begin{abstract}
Almost all proposed approaches to nonlinear receding horizon control with guaranteed stability are based on adding stabilizing constraints, using linearizations of the system, or knowing an upper bound on the value function of an infinite horizon optimal control problem. In this contribution, we present a new approach using none of these ingredients. The idea is to use a dynamic receding horizon controller, where the dynamic part is introduced to tune emphasis between short-range optimality and stability. The result is a design procedure applicable to a nontrivial class of unconstrained nonlinear systems.
\end{abstract}

Keywords: Predictive Control, Optimal Control, Stabilization of Non-linear Systems 


\title{
NONLINEAR RECEDING HORIZON CONTROL: STABILITY WITHOUT STABILIZING CONSTRAINTS
}

\author{
Johan Löfberg \\ Division of Automatic Control, \\ Department of Electrical Engineering, \\ Linköpings universitet, \\ SE-581 83 Linköping, Sweden. \\ fax: $\quad+4613282622$ \\ e-mail: johanleisy.liu.se
}

Keywords: Predictive Control, Optimal Control, Stabilization of Non-linear Systems.

\begin{abstract}
Almost all proposed approaches to nonlinear receding horizon control with guaranteed stability are based on adding stabilizing constraints, using linearizations of the system, or knowing an upper bound on the value function of an infinite horizon optimal control problem. In this contribution, we present a new approach using none of these ingredients. The idea is to use a dynamic receding horizon controller, where the dynamic part is introduced to tune emphasis between short-range optimality and stability. The result is a design procedure applicable to a nontrivial class of unconstrained nonlinear systems.
\end{abstract}

\section{Introduction}

Receding horizon control (RHC), also known as model predictive control, is a control paradigm based on solving optimal control problems numerically on-line. Since the derivation of an optimal feedback law is intractable for most nonlinear systems, RHC is almost the only constructive method to do optimal control for nonlinear systems.

A fundamental problem with RHC is the lack of guaranteed closed loop stability. Stability can be lost since the numerical solution of the optimal control problem has to be solved over a finite horizon, and this can lead to shortsightedness of the controller.

In this paper, we address the stability issue in RHC for nonlinear systems and develop a new approach to design globally stabilizing RHC controllers. In contrast to the majority of existing methods to guarantee stability, we do not resort to arguments using linearizations or by adding stabilizing constraints to the underlying optimal control problem.

\section{Receding horizon control}

Putting technical conditions aside for the moment, assume we have a nonlinear system

$$
\dot{x}(t)=f(x(t), u(t))
$$

and our goal is to find the feedback law that at every timeinstant minimizes the infinite horizon performance measure

$$
J_{\infty}\left(x(t), u_{t}\right)=\int_{t}^{\infty} \ell\left(x_{t}(\tau), u_{t}(\tau)\right) d \tau
$$

In the expression above, we introduced the control function $u_{t}(\tau), \tau \in[t, \infty)$ defined at time $t$. The control function gives a trajectory $x_{t}(\tau)$ which satisfies the system dynamics $\dot{x}_{t}(\tau)=f\left(x_{t}(\tau), u_{t}(\tau)\right)$ and the initial condition $x_{t}(t)=x(t)$.

Finding the optimal feedback law is in general an intractable problem since it requires the solution of a nonlinear partial differential equation, the Hamilton-Jacobi equation. It was early recognized [9] that a natural way to overcome this is to resort to a numerical solution, i.e. to continuously minimize the integral on-line. In order to do this, the infinite horizon has to be truncated. The performance measure used on-line will then be

$$
J\left(x(t), u_{t}\right)=\int_{t}^{t+T} \ell\left(x_{t}(\tau), u_{t}(\tau)\right) d \tau
$$

and give us an optimal control sequence to be used over the horizon ( $*$ will be used throughout this paper to denote optimality in an optimal control problem)

$$
u_{t}^{*}=\arg \min _{u_{t}} J\left(x(t), u_{t}\right)
$$

Receding horizon control means that only the first portion of the optimal solution is used, and the problem is solved again a short time-instant later. Implicitly, this will define the feedback law $u(t)=u_{t}^{*}(t)$.

Although this might seem like a simple and sound way to define a feedback law, it turns out that the control law can destabilize the system. The reason can intuitively be explained as a consequence of the introduced shortsightedness of the controller.

\subsection{Stability of RHC, previous work}

The first step towards a stabilizing scheme is to add a terminal state weight

$J\left(x(t), u_{t}\right)=\int_{t}^{t+T} \ell\left(x_{t}(\tau), u_{t}(\tau)\right) d \tau+V\left(x_{t}(t+T)\right)$ 
It is fairly easy to derive sufficient conditions on the weight $V(x)$ to guarantee stability. A typical condition is that there should exist a stabilizing feedback $k(x)$ such that

$$
\dot{V}(x, k(x))+\ell(x, k(x)) \leq 0
$$

By integrating this expression, we see that it means that $V(x(t))$ is an upper bound of the infinite horizon cost when $u(t)=k(x(t))$ is used, $J_{\infty}(x(t), k(x(\cdot)))$.

The condition might seem simple at a first glance, but derivation of a terminal state weight $V$ such that (6) holds is actually a major problem.

A simple and often used approach to guarantee stability is to add a terminal state constraint $x_{t}^{*}(t+T)=0$. This can intuitively be interpreted as a terminal state weight which is infinite everywhere except in the origin. This approach was proposed, in the context of linear continuous-time systems, in [8] and later generalized to nonlinear systems and thoroughly analyzed in, e.g., [10]. In [13], the terminal state constraint was relaxed for linear systems by having a terminal state constraint on only the unstable modes. The stable modes were addressed by adding a terminal state weight on these, satisfying (6) for $k(x)=0$.

The idea with a terminal state weight was taken further in [2], now considering also nonlinear systems. The main idea, generalizing ideas in $[11,13]$ was to use a terminal state inequality $x_{t}^{*}(t+T) \in \Omega$. The ellipsoidal set $\Omega$, a linear controller $k(x)$ and a quadratic terminal state weight satisfying (6) in $\Omega$, were all designed using arguments involving the linearized system. [2] also proposed a more general scheme using a nonlinear controller $k(x)$ and a terminal state weight satisfying (6) in a terminal set $\Omega$. These ideas have recently been generalized and studied in a control Lyapunov function framework (defined below) $[12,4,3]$. However, in the design procedure one still has to find a terminal state weight globally satisfying (6), or resorting to arguments based on linearizations and/or various state constraints.

\section{Main result}

We see from the quick review above that there are three main ideas in the available approaches; assuming knowledge of a terminal state weight globally satisfying (6), using arguments based on the linearized system and adding stabilizing constraints. Our goal in this paper is to develop a method that use neither of these ingredients.

To begin with, we assume we have a global control Lyapunov function $V(x)$ and corresponding controller $k(x)$ [6]

Definition $1 A$ smooth positive definite and radially unbounded function $V: \mathbf{R}^{n} \rightarrow \mathbf{R}_{+}$is called a control Lyapunov function (CLF) if there exist a control law $u=k(x)$ such that

$$
\dot{V}(x, k(x))=V_{x} f(x, k(x))<0, \quad \forall x \neq 0
$$

Notice the small but very important difference in the requirement on the CLF compared to the condition (6). Of course, knowledge of a CLF is an essential limitation, but with recent developments in nonlinear control there are many methods available for designing these, such as backstepping, feedforwarding and exact linearization.

The requirement (6) shows up in stability analysis of RHC as a sufficient condition. To be precise, the condition is

$$
\begin{aligned}
\dot{V}\left(x_{t}^{*}(t+T), k\left(x_{t}^{*}(t+T)\right)\right) & \\
& +\ell\left(x_{t}^{*}(t+T), k\left(x_{t}^{*}(t+T)\right)\right) \leq 0
\end{aligned}
$$

Our method is based on the idea that the condition above can be achieved, at-least intuitively, by multiplying $\ell$ with some sufficiently small constant $\lambda$, since we know from the design that $\dot{V}(x, k(x))<0$ and $\ell(x, k(x))>0$. When we use this new weight, we get the constraint

$$
\begin{aligned}
\dot{V}\left(x_{t}^{*}(t+T), k\left(x_{t}^{*}(t+T)\right)\right) & \\
& +\lambda \ell\left(x_{t}^{*}(t+T), k\left(x_{t}^{*}(t+T)\right)\right) \leq 0
\end{aligned}
$$

If this still does not hold, $\lambda$ should be decreased. This motivates introduction of a new state $\lambda(t)$, which can be thought of as a state in the RHC controller, with dynamics

$$
\begin{aligned}
\dot{\lambda}(t) \sim-\lambda(t) \ell\left(x_{t}^{*}(t+\right. & \left.T), k\left(x_{t}^{*}(t+T)\right)\right) \\
& -\dot{V}\left(x_{t}^{*}(t+T), k\left(x_{t}^{*}(t+T)\right)\right)
\end{aligned}
$$

After introducing this state, we define a new optimal control problem to be used in the RHC controller

$$
\begin{aligned}
& J\left(x(t), u_{t}, \lambda(t)\right)= \\
& \quad \lambda(t) \int_{t}^{t+T} \ell\left(x_{t}(\tau), u_{t}(\tau)\right) d \tau+V\left(x_{t}(t+T)\right)
\end{aligned}
$$

The problem is now to find an update-law for $\lambda(t)$ such that the RHC scheme will guarantee stability. To find such an update, we first have to introduce some assumptions on the system and design variables

A1. $f(0,0)=0, f(x, u)$ continuously differentiable.

A2. $\ell(x, u)>0 \forall(x, u) \neq 0, \ell(0,0)=0$. Furthermore, $\ell(x, u)$ is two times continuously differentiable and for simplicity it is assumed that the mixed derivative $\ell_{x u}=0$.

A3. $V(x)$ is a global CLF with the continuously differentiable controller $k(x)$. Furthermore, the linearization of the system $\dot{x}=f(x, k(x))$ is asymptotically stable.

A4. $\int_{t}^{t+T} \ell\left(x_{t}(\tau), u_{t}(\tau)\right) d \tau \rightarrow \infty$ when $\|x(t)\| \rightarrow \infty$ for any $u_{t}(\tau)$.

A5. There exist a minimizing argument $u_{t}^{*}$ of (10) for all $x(t)$ and $\lambda(t)>0$.

A6. $J\left(x(t), u_{t}^{*}, \lambda(t)\right)$ is continuously differentiable when $u(t)$ and $\dot{\lambda}(t)$ are chosen according to Theorem 1 
The assumptions stated are those needed to prove that $J\left(x(t), u_{t}^{*}, \lambda(t)\right)$ can be used as a Lyapunov function. The assumption on existence of a solution A5 implicitly impose additional constraints on $f(x, u), \ell(x, u)$ and $V(x)$. Typical results can be found in, e.g.,Theorem 6.2 in [1]. Admittedly, Assumption 6 is hard to verify. The reason for assuming this is to obtain an intuitive result using standard Lyapunov theory. For some additional discussion on the assumptions, see the proof and remarks in the appendix.

With the introduced assumptions, we state our main result.

Theorem 1 (Stabilizing nonlinear RHC) Suppose (A1-A6) are satisfied and $\lambda(0)>0$. Then the following RHC scheme is globally asymptotically stabilizing (we use the notation $e^{*}=x_{t}^{*}(t+T)$ to save space $)$

$$
\begin{aligned}
u_{t}^{*} & =\arg \min _{u_{t}} J\left(x(t), u_{t}, \lambda(t)\right) \\
u(t) & =u_{t}^{*}(t) \\
\dot{\lambda}(t) & =\frac{-\lambda(t) \ell\left(e^{*}, k\left(e^{*}\right)\right)-\dot{V}\left(e^{*}, k\left(e^{*}\right)\right)}{\int_{t}^{t+T} \ell\left(x_{t}^{*}(\tau), u_{t}^{*}(\tau)\right) d \tau}
\end{aligned}
$$

Proof: See the appendix.

We notice that the complexity of the optimal control problem is the same as for the original problem, i.e. stability is not achieved at the expense of a more complicated optimization. Moreover, the right-hand side of the differential equation that controls $\lambda(t)$ is easily calculated since both the numerator and denominator can be obtained from the solution of the optimization problem.

\section{Numerical example}

The system we analyze is taken from [7]. The model is in principle a simple description of a jet engine compressor (modulo some transformations), but in this example we disregard any physical considerations.

$$
\begin{aligned}
& \dot{x}_{1}=-\frac{3}{2} x_{1}^{2}-\frac{1}{2} x_{1}^{3}-x_{2} \\
& \dot{x}_{2}=u
\end{aligned}
$$

In [7], lean backstepping is applied to derive the control law $k(x)=K x, K=\left[k_{1}-k_{2}\right], k_{1}=\left(c_{1}+\frac{9}{8}\right) k_{2}, k_{2}=c_{1}+c_{2}+\frac{9}{8}$. The design parameters for the controller are $c_{1}>0$ and $c_{2}>$ 0 . The following CLF is obtained when the feedback law is developed $\left(c_{0}=c_{1}+\frac{9}{8}\right)$.

$V(x)=c_{0}\left(\left(\frac{c_{1}}{2}+\frac{9}{16}\right) x_{1}^{2}+\frac{1}{2} x_{1}^{3}+\frac{1}{8} x_{1}^{4}\right)+\frac{1}{2}\left(x_{2}-c_{0} x_{1}\right)^{2}$

This CLF can be shown to fulfill

$$
\dot{V}(x, K x) \leq-x^{T} W x
$$

where

$$
W=\left[\begin{array}{cc}
c_{0} c_{1}^{2}+c_{2} c_{0}^{2} & -c_{0} c_{2} \\
-c_{0} c_{2} & c_{2}
\end{array}\right] \succ 0
$$

We choose to optimize a standard quadratic performance measure $\ell(x, u)=x^{T} Q x+u^{T} R u$ with $Q=I$ and $R=1$. Rather arbitrarily, we pick $c_{1}=c_{2}=1$ to create $k(x)$.

The initial state in the simulation was $\left[\begin{array}{ll}-2 & 1\end{array}\right]^{T}$. The motivation to study this initial condition is that $x_{1}=-2$ is the position where the nonlinearity in the systems is most destabilizing. The prediction horizon $T$ was $0.5 \mathrm{sec}$. The proposed RHC algorithm was applied and Figure 1 shows how $\lambda(t)$ evolved. We see that $\lambda(t)$ changes rapidly in the beginning. If this is

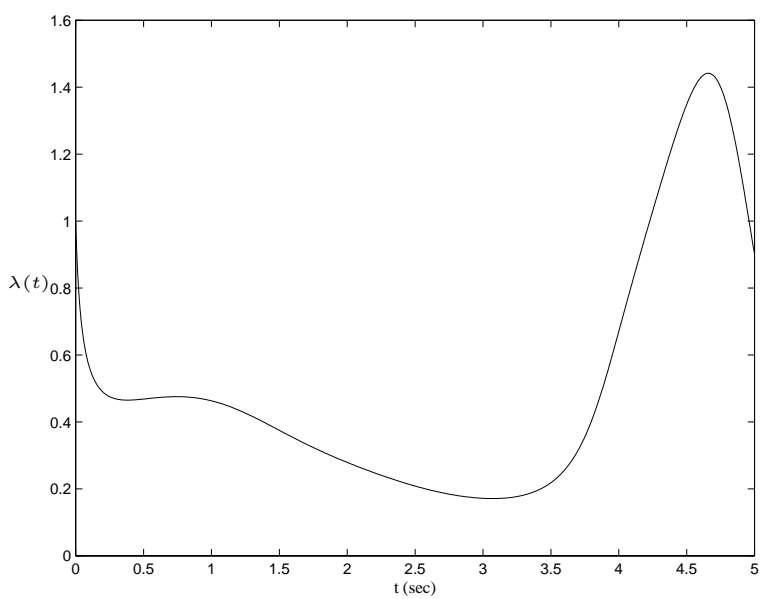

Figure 1: Response of the dynamic weighting $\lambda(t)$.

a problem, this behavior can in some cases be improved upon by using the extension discussed in Remark 3 in the appendix. The state and control trajectories are shown in Figure 2.

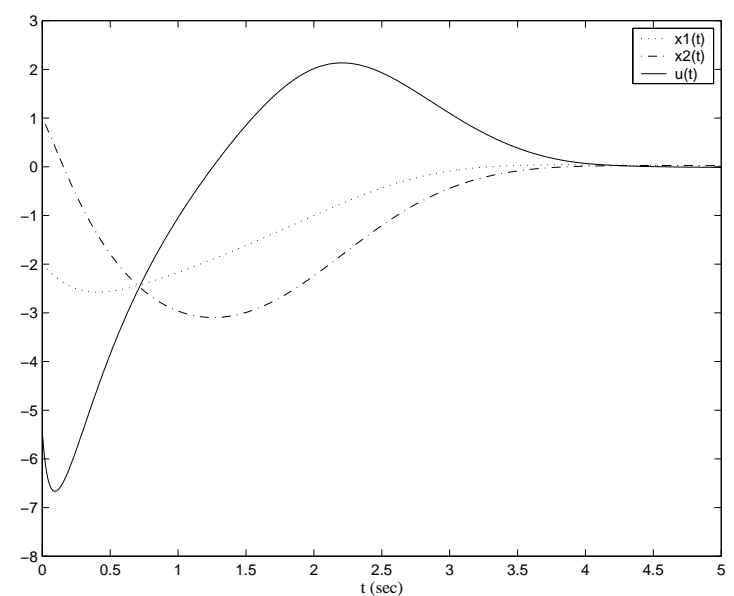

Figure 2: State and control trajectories.

The original infinite horizon cost $J_{\infty}$ for the proposed controller turned out to be 46 . The fact that we have a linear controller $k(x)$, a quadratic performance measure and upper bound bound on $\dot{V}(x, K x)$ makes it possible to easily calculate a constant $\lambda(t)$ so that (8) is globally satisfied. Inserting the performance measure and the upper bound on $\dot{V}(x, k(x))$ in (8) gives us a sufficient constraint for stability

$$
-x^{T} W x+\lambda(t) x^{T}\left(Q+K^{T} R K\right) x \leq 0
$$


This condition holds for all $x$ if $\lambda(t)$ is chosen as a constant smaller than the smallest eigenvalue of $\left(Q+K^{T} R K\right)^{-1} W$. For the parameters used in this example, we find that stability is guaranteed if we fix $\lambda(t)=0.09$. This choice was used in a RHC controller and gave $J_{\infty}=66$. An intuitive explanation for the detoriated performance is that this tuning puts too much emphasis on stability, hence our dynamic choice of $\lambda(t)$ is indeed beneficial. Finally, the cost using the nominal controller $k(x)$ was also calculated and gave $J_{\infty}=77$.

\section{Conclusion}

A new approach to stabilizing receding horizon control has been introduced. Stability is obtained by using a dynamic controller, where the dynamics is used to tune emphasis between stability and short-range optimality by adapting the terminal state weight. We do not claim that the approach is suitable for arbitrary nonlinear systems, but we hope that the method can spark interest towards the development of RHC schemes where stability is taken care of without the use of artificially introduced stabilizing constraints.

The approach is currently solely aimed at guaranteed stability, i.e., performance is not addressed. An important extension is to study how the degree of freedom available in the update-law of $\lambda(t)$ can be exploited in order to improve performance.

There is still a lot of work that can be done in order to improve the presented result. Removing the assumption on differentiability of the optimal cost, and the various assumptions on the performance measure and the system are topics that deserve a closer look.

\section{References}

[1] L.D. Berkovitz. Optimal Control Theory. Applied Mathematical Sciences. Springer-Verlag, 1974.

[2] H. Chen. Stability and Robustness Considerations in Nonlinear Model Predictive Control. PhD thesis, Universität Stuttgart, 1997.

[3] F.A.C.C. Fontes. A general framework to design stabilizing nonlinear model predictive controllers. Systems \& Control Letters, 42:127-143, 2001.

[4] A. Jadbabaie. Receding Horizon Control of Nonlinear Systems: A Control Lyapunov Function Approach. PhD thesis, California Institute of Technonlogy, Pasadena, California, 2000.

[5] H. Khalil. Nonlinear Systems. Macmillan Publishing Company, 1992.

[6] M. Krstić, I. Kanellakopoulos, and P. Kokotović. Nonlinear and Adaptive Control Design. Adaptive and Learning Systems for Signal Processing, Communications, and Control. John Wiley \& Sons, 1995.
[7] M. Krstić and P. Kokotović. Lean backstepping design for a jet engine compressor model. In Proceedings of the 4th IEEE Conference on Control Applications, pages 1047$1052,1995$.

[8] W.H. Kwon and A.E. Pearson. A modified quadratic cost problem and feedback stabilization of a linear system. IEEE Transactions on Automatic Control, 22(5):838$842,1977$.

[9] E.B. Lee and L. Markus. Foundations of Optimal Control Theory. The SIAM Series in Applied Mathematics. John Wiley \& Sons, 1968.

[10] D.Q. Mayne and H. Michalska. Receding horizon control of nonlinear systems. IEEE Transactions on Automatic Control, 35(7):814-824, 1990.

[11] H. Michalska and D.Q. Mayne. Robust receding horizon control of constrained nonlinear systems. IEEE Transactions on Automatic Control, 38(1):1623-1633, 1993.

[12] J.A. Primbs. Nonlinear Optimal Control: A Receding Horizon Approach. $\mathrm{PhD}$ thesis, California Institute of Technonlogy, Pasadena, California, 1999.

[13] J.B. Rawlings and K.R. Muske. The stability of constrained receding horizon control. IEEE Transactions on Automatic Control, 38(10):1512-1516, 1993.

\section{A Proof of Theorem 1}

The idea in the proof is to use $J\left(x(t), u_{t}^{*}, \lambda(t)\right)$, from now on called $J^{*}(t)$, as a Lyapunov function.

Our first goal is to derive an expression containing $J^{*}(t+\delta)$, i.e., the optimal cost at some future time instant. This will used to find the derivative of our proposed Lyapunov function. We will then show that $J^{*}(t)$ satisfies conditions necessary to be used a a Lyapunov function.

To begin with, we add and subtract some terms to the optimal cost

$$
\begin{aligned}
J^{*}(t)= & \lambda(t) \int_{t}^{t+T} \ell\left(x_{t}^{*}(\tau), u_{t}^{*}(\tau)\right) d \tau+V\left(x_{t}^{*}(t+T)\right) \\
& +\lambda(t+\delta) \int_{t+T}^{t+T+\delta} \ell\left(\tilde{x}_{t}(\tau), \tilde{u}_{t}(\tau)\right) d \tau \\
& -\lambda(t+\delta) \int_{t+T}^{t+T+\delta} \ell\left(\tilde{x}_{t}(\tau), \tilde{u}_{t}(\tau)\right) d \tau \\
& +V\left(\tilde{x}_{t}(t+T+\delta)\right)-V\left(\tilde{x}_{t}(t+T+\delta)\right)
\end{aligned}
$$

In the expression above, we introduced two new variables, $\tilde{u}_{t}$ and $\tilde{x}_{t}$. The first one, $\tilde{u}_{t}$, is an arbitrary choice of control over the new segment $[t+T, t+T+\delta]$. The state trajectory this control gives is denoted $\tilde{x}_{t}$

$$
\dot{\tilde{x}}_{t}(\tau)=f\left(\tilde{x}_{t}(\tau), \tilde{u}_{t}(\tau)\right), \quad \tilde{x}_{t}(t+T)=x_{t}^{*}(t+T)
$$


We split the integral $\int_{t}^{t+T}(\cdot) d \tau$ into $\int_{t}^{t+\delta}(\cdot) d \tau+\int_{t+\delta}^{t+T}(\cdot) d \tau$. Furthermore, we replace $\lambda(t)$ with $\lambda(t)+\lambda(t+\delta)-\lambda(t+\delta)$. With these manipulations, we write the first term in (15) as

$$
\begin{aligned}
\lambda(t) \int_{t}^{t+T} \ell\left(x_{t}^{*}(\tau), u_{t}^{*}(\tau)\right) d \tau= \\
\lambda(t) \int_{t}^{t+\delta} \ell\left(x_{t}^{*}(\tau), u_{t}^{*}(\tau)\right) d \tau \\
+\lambda(t+\delta) \int_{t+\delta}^{t+T} \ell\left(x_{t}^{*}(\tau), u_{t}^{*}(\tau)\right) d \tau \\
+(\lambda(t)-\lambda(t+\delta)) \int_{t+\delta}^{t+T} \ell\left(x_{t}^{*}(\tau), u_{t}^{*}(\tau)\right) d \tau
\end{aligned}
$$

We plug this expression into (15) and sort the expression to have terms involving $t+\delta$ first

$$
\begin{gathered}
J^{*}(t)=\lambda(t+\delta) \int_{t+\delta}^{t+T} \ell\left(x_{t}^{*}(\tau), u_{t}^{*}(\tau)\right) d \tau \\
+\lambda(t+\delta) \int_{t+T}^{t+T+\delta} \ell\left(\tilde{x}_{t}(\tau), \tilde{u}_{t}(\tau)\right) d \tau+V\left(\tilde{x}_{t}(t+T+\delta)\right) \\
\quad-\lambda(t+\delta) \int_{t+T}^{t+T+\delta} \ell\left(\tilde{x}_{t}(\tau), \tilde{u}_{t}(\tau)\right) d \tau \\
+\lambda(t) \int_{t}^{t+\delta} \ell\left(x_{t}^{*}(\tau), u_{t}^{*}(\tau)\right) d \tau \\
+(\lambda(t)-\lambda(t+\delta)) \int_{t+\delta}^{t+T} \ell\left(x_{t}^{*}(\tau), u_{t}^{*}(\tau)\right) d \tau \\
-V\left(\tilde{x}_{t}(t+T+\delta)\right)+V\left(x_{t}^{*}(t+T)\right)
\end{gathered}
$$

The first two rows in the expression above is the cost at time $t+\delta$, using the old optimal $u_{t}^{*}$ over the horizon $[t+\delta, T]$ and $\tilde{u}_{t}$ over the new horizon $[t+T, t+T+\delta]$. Clearly, this cost cannot be lower than the optimal cost, hence

$$
\begin{gathered}
J^{*}(t) \geq J^{*}(t+\delta) \\
-\lambda(t+\delta) \int_{t+T}^{t+T+\delta} \ell\left(\tilde{x}_{t}(\tau), \tilde{u}_{t}(\tau)\right) d \tau \\
+\lambda(t) \int_{t}^{t+\delta} \ell\left(x_{t}^{*}(\tau), u_{t}^{*}(\tau)\right) d \tau \\
+(\lambda(t)-\lambda(t+\delta)) \int_{t+\delta}^{t+T} \ell\left(x_{t}^{*}(\tau), u_{t}^{*}(\tau)\right) d \tau \\
\quad-V\left(\tilde{x}_{t}(t+T+\delta)\right)+V\left(x_{t}^{*}(t+T)\right)
\end{gathered}
$$

Up to now, we have only made some algebraic manipulations, but at this point, we have to make a crucial decision on how to proceed. One approach could be to continue without assuming differentiability of the involved expressions and instead look at the sequence $J^{*}(t)$ [3]. However, to obtain simple and intuitive result, we assume $J^{*}(t)$ to be continuously differentiable.
Standard arguments with $\lim _{\delta \rightarrow 0^{+}}$yields

$$
\begin{aligned}
& \dot{J}^{*}(t) \leq-\lambda(t) \ell\left(x_{t}^{*}(t), u_{t}^{*}(t)\right) \\
& +\lambda(t) \ell\left(x_{t}^{*}(t+T), \tilde{u}_{t}(t+T)\right) \\
& +\dot{\lambda}(t) \int_{t}^{t+T} \quad \ell\left(x_{t}^{*}(\tau), u_{t}^{*}(\tau)\right) d \tau \\
& \quad+\dot{V}\left(x_{t}^{*}(t+T), \tilde{u}_{t}(t+T)\right)
\end{aligned}
$$

Since our ultimate goal is to show that $J^{*}(t)$ is a Lyapunov function, we would like the right hand side of the above expression to be negative. From the assumption on the cost, $-\lambda(t) \ell\left(x_{t}^{*}(t), u_{t}^{*}(t)\right.$ is negative if $\lambda(t)>0$. For reasons that will be clear later, we chose $\tilde{u}_{t}(t+T)=k\left(x_{t}^{*}(t+T)\right)$. By looking at (17), we see that if

$$
\begin{aligned}
\lambda(t) \ell\left(x_{t}^{*}(t+T),\right. & \left.k\left(x_{t}^{*}(t+T)\right)\right) \\
& +\dot{\lambda}(t) \int_{t}^{t+T} \ell\left(x_{t}^{*}(\tau), u_{t}^{*}(\tau)\right) d \tau \\
& +\dot{V}\left(x_{t}^{*}(t+T), k\left(x_{t}^{*}(t+T)\right)\right) \leq 0
\end{aligned}
$$

we would have

$$
\dot{J}^{*}(t) \leq-\lambda(t) \ell\left(x_{t}^{*}(t), u_{t}^{*}(t)\right)
$$

Condition (18) thus motives the proposed update-law (with $e^{*}$ denoting $x_{t}^{*}(t+T)$ to save space)

$$
\dot{\lambda}(t)=\frac{-\lambda(t) \ell\left(e^{*}, k\left(e^{*}\right)\right)-\dot{V}\left(e_{t}^{*}, k\left(e^{*}\right)\right)}{\int_{t}^{t+T} \ell\left(x_{t}^{*}(\tau), u_{t}^{*}(\tau)\right) d \tau}
$$

Before we proceed to prove stability, we need some supporting results. To begin with, we see from the definition of $\dot{\lambda}(t)$ that $\lambda(t)$ will remain positive (assuming $\lambda(0)>0$ ) since $-\dot{V}(x, k(x))$ and the denominator in (20) are both positive definite. To prove stability, it is crucial to show that $\lambda(t)$ is not only positive, but also bounded from below. To do this, we first need a bound on $x^{*}(t+T)$.

We know that there exist a solution to the optimal control problem, hence $J^{*}(0)$ is bounded. This immediately implies $V\left(x^{*}(0+T)\right) \leq J^{*}(0)<\infty$. Now, construct a ball $\|x\| \leq r_{1}$ containing the level-sets $V(x) \leq J^{*}(0)$. From (19), we know that $J^{*}(t)$ is non-increasing since $\lambda(t)$ is positive and $\ell$ is positive definite. Hence, we know that $J^{*}(t) \leq J^{*}(0)$ which implies that $\left\|x^{*}(t+T)\right\| \leq r_{1}$.

From (20), we see that $\dot{\lambda}(t)$ is non-negative when

$$
\lambda(t) \leq \frac{-\dot{V}\left(x_{t}^{*}(t+T), k\left(x_{t}^{*}(t+T)\right)\right)}{\ell\left(x_{t}^{*}(t+T), k\left(x_{t}^{*}(t+T)\right)\right)}
$$

We now select constant $0<r_{2}<r_{1}$, where $r_{2}$ will be chosen later, and look at the minimum of the right-hand side of (21) in the set $r_{2} \leq\left\|x^{*}(t+T)\right\| \leq r_{1}$

$$
\min _{r_{2} \leq\|x\| \leq r_{1}} \frac{-\dot{V}(x, k(x))}{\ell(x, k(x))}=\gamma_{1}>0
$$


This follows from the assumption of continuity and positive definiteness on $\ell$ and $-\dot{V}$. From this we find that $\lambda(t)$ increases when it falls below $\gamma_{1}$ and $r_{2} \leq\left\|x^{*}(t+T)\right\| \leq r_{1}$.

We now employ a somewhat different approach to find

$$
\min _{\|x\| \leq r_{2}} \frac{-\dot{V}(x, k(x))}{\ell(x, k(x))}
$$

From the assumptions, the following models hold around the origin

$$
\begin{aligned}
\dot{x} & =(A+B K) x+o(\|x\|) \\
V(x) & =x^{T} P x+o\left(\|x\|^{2}\right) \\
\ell(x, k(x)) & =x^{T} Q x+x^{T} K^{T} R K x+o\left(\|x\|^{2}\right)
\end{aligned}
$$

where

$$
\begin{array}{r}
A=f_{x}(0,0), B=f_{u}(0,0), K=k_{x}(0) \\
P=\frac{1}{2} V_{x x}(0,0), Q=\frac{1}{2} \ell_{x x}(0,0), R=\frac{1}{2} \ell_{u u}(0,0)
\end{array}
$$

Inserting these models yields

$$
\min _{\|x\| \leq r_{2}} \frac{-x\left((A+B K)^{T} P+P(A+B K)\right) x+g_{1}(x)}{x^{T}\left(Q+K^{T} R K\right) x+g_{2}(x)}
$$

where $g_{1}(x)$ and $g_{2}(x)$ collect higher order terms

$$
\begin{aligned}
& \frac{\left\|g_{1}(x)\right\|}{\|x\|^{2}} \leq \rho_{1}(x), \lim _{x \rightarrow 0} \rho_{1}(x)=0 \\
& \frac{\left\|g_{2}(x)\right\|}{\|x\|^{2}} \leq \rho_{2}(x), \lim _{x \rightarrow 0} \rho_{2}(x)=0
\end{aligned}
$$

From Assumption 3 we know that there exist a positive definite matrix $W$ such that

$$
(A+B K)^{T} P+P(A+B K)=-W
$$

Inserting this and the higher order terms, we obtain a lower bound by looking at the conservative approximation

$$
\min _{\|x\| \leq r_{2}} \frac{x^{T}\left(W-\rho_{1}(x) I\right) x}{x^{T}\left(Q+K^{T} R K+\rho_{2}(x) I\right) x}
$$

Since $\rho_{1}(x)$ tends to zero, we can always find an $r_{2}$ such that $\|x\| \leq r_{2}$ guarantees, say, $\rho_{1}(x) \leq \frac{1}{2} \nu$ where $\nu$ denotes the smallest eigenvalue of $W$. For $\|x\| \leq r_{2}$ we thus have $W-$ $\rho(x) I \succeq \frac{1}{2} W$. Furthermore, let

$$
0 \leq \alpha=\max _{\|x\| \leq r_{2}} \rho_{2}(x)<\infty
$$

With these bounds on $\rho_{1}(x)$ and $\rho_{2}(x)$ we obtain an even more conservative approximation by solving

$$
\min _{\|x\| \leq r_{2}} \frac{\frac{1}{2} x^{T} W x}{x^{T}\left(Q+K^{T} R K+\alpha I\right) x}
$$

Since $Q$ and $R$ are positive definite, we find that the minimum of this expression is equal to the smallest eigenvalue of the matrix $\frac{1}{2}\left(Q+K^{T} R K+\alpha I\right)^{-1} W$. Let $\gamma_{2}$ denote this lower bound.
We can now conclude that for $\left\|x^{*}(t+T)\right\| \leq r_{2}, \lambda(t)$ has to start to increase if it is smaller than $\gamma_{2}$.

Combining the two bounds, we find that $\dot{\lambda}(t)$ is non-negative when $\lambda(t) \leq \min \left(\gamma_{1}, \gamma_{2}\right)$. This immediately implies that $\lambda(t) \geq \min \left(\lambda(0), \gamma_{1}, \gamma_{2}\right)$. With this, we are ready to employ the Barbashin-Krasovskii theorem [5].

Theorem 2 Let $x=0$ be an equilibrium point and the continuously differentiable function $V: \mathbf{R}^{n} \rightarrow \mathbf{R}_{+}$be positive definite and radially unbounded with

$$
\dot{V}(x)<0, \quad \forall x \neq 0
$$

then $x=0$ is globally asymptotically stable

To begin with, we assumed $J^{*}(t)$ to be continuously differentiable. It is easy to realize that $J^{*}(t)=0$ if $x(t)=0$. Moreover, since $\lambda(t)$ is bounded from below, we know that $J^{*}(t)>0 \forall x \neq 0$. Assumption 4 ensures, once again relying on $\lambda(t)$ being bounded from below, that $J^{*}(t) \rightarrow \infty$ when $\|x(t)\| \rightarrow \infty$. Finally, $\dot{J}^{*}(t)<0 \forall x \neq 0$ according to (19) and positivity on $\lambda(t)$. Hence we are able to conclude that $x(t)$ is asymptotically stable.

Remark 1 Notice that the linearized system is not used for the controller design, it is only used in the stability proof.

Remark 2 A second remark that concerns the assumption on local behavior of $f(x, k(x)), \dot{V}(x, k(x))$ and $\ell(x, k(x))$. These assumptions are only used to show that the quotient

$$
\frac{-\dot{V}(x, k(x))}{\ell(x, k(x))}
$$

is bounded from below in the origin. Clearly, the assumed local properties are not necessary for this to hold.

Remark 3 The update-law for $\lambda(t)$ is of course not unique. There are many alternatives that might work just as well, or even better. One such choice is $(0 \leq \epsilon<1)$

$\dot{\lambda}(t)=\frac{-\lambda(t) \ell\left(e^{*}, k\left(e^{*}\right)\right)-\dot{V}\left(e_{t}^{*}, k\left(e^{*}\right)\right)+\epsilon \lambda(t) \ell\left(x(t), u_{t}^{*}(t)\right)}{\int_{t}^{t+T} \ell\left(x_{t}^{*}(\tau), u_{t}^{*}(\tau)\right) d \tau}$ 